\title{
Teneurs en minéraux des fourrages de chaume et de leurs adventices : l'exemple d'une exploitation agricole du sud-est de la France (Vaucluse)
}

\author{
Eric Gerbaud ${ }^{\mathrm{a}}$, Thierry DutoIT ${ }^{\mathrm{a} *}$, Aurélia BARROIT ${ }^{\mathrm{a}}$, \\ Bernard TOUSSAINT ${ }^{b}$ \\ a Institut Méditerranéen d'Écologie et de Paléoécologie, Université de Provence, \\ UMR/CNRS 6116 IMEP, FST Saint-Jérôme, Case 461, 13397 Marseille Cedex 20, France \\ ${ }^{\mathrm{b}}$ Laboratoire d'Écologie des Prairies, Université Catholique de Louvain, \\ 6600 Michamps, Bastogne, Belgique
}

(Reçu le 21 mars 2001 ; accepté le 22 novembre 2001)

\begin{abstract}
Mineral contents of cereal stubble: the case of an agricultural exploitation in southeastern France (Vaucluse). In south-eastern France, where extensive farming systems still exists, including itinerant livestock keeping, cereal stubbles are used at the end of the grazing season. Nevertheless, little information on the mineral values of this forage is available. For the same grazing system of an agricultural exploitation of the Luberon regional natural park (south-eastern France, Vaucluse), mineral analyses of forage samples of four contrasting ecosystems (a fallow-land, a dry grassland, a wet meadow and a cereal stubble) show that the last ecosystem presents a dry biomass significantly lower than the three other plots sampled from July to October 1999. Nevertheless, the forage value of stubble seems to be of interest during the summer because the results show significant low concentrations of cellulose and insoluble ash. In contrast, the forage sampled in stubble has significantly higher concentrations of crude protein, $\mathrm{Ca}, \mathrm{Mg}$ and $\mathrm{K}$.
\end{abstract}

conservation / arable weeds / sheep grazing / mineral content / extensive agriculture

Résumé — Dans le sud-est de la France, où persistent des exploitations agricoles extensives incluant l'élevage ovin de parcours, les fourrages issus de champs de céréales moissonnés (chaumes et adventices) permettent le bouclage de la saison de pâturage. En revanche, peu de données sont disponibles sur la valeur nutritive de ces fourrages (teneurs en minéraux). Dans une exploitation agricole représentative des pratiques menées dans la majorité de celles du territoire du Parc naturel régional du Luberon (Vaucluse), les teneurs en minéraux de fourrages échantillonnés dans trois parcelles écologiquement contrastées (prairie humide, friche post-culturale, pelouse sèche) et une parcelle moissonnée ont été dosées. Nos résultats montrent que le fourrage échantillonné dans la parcelle moissonnée présente une biomasse sèche significativement inférieure à celle des trois autres parcelles entre juillet

* Correspondance et tirés-à-part

Tél. : 33491288732 ; fax : 33491288051 ; e-mail : thierry.dutoit @ univ.u-3mrs.fr 
et octobre 1999. Cependant la valeur nutritive du fourrage de chaumes avec les adventices apparait très intéressante en période estivale car les teneurs en cellulose et cendres insolubles sont significativement inférieures. Au contraire, pour les matières azotées totales et les éléments calcium, magnésium et potassium, ses teneurs sont significativement supérieures.

conservation / adventices / pâturage ovin / teneur minérale / agriculture extensive

\section{INTRODUCTION}

De nombreuses recherches ont été menées sur la dynamique des mauvaises herbes des grandes cultures [15, 17]. Si la majorité des travaux ont été réalisés en malherbologie, certains chercheurs ont cependant constaté, dès les années 1960, la régression de beaucoup d'espèces, [3-5] en liaison avec l'accroissement de 1'application d'engrais azotés et de la généralisation des traitements herbicides [1, 2, 28, 33, 36].

Depuis quelques années, un certain nombre de ces recherches ont ainsi eu pour objectifs la création d'une lutte intégrée qui se traduit en malherbologie par l'application d'herbicides plus sélectifs, de nouvelles façons culturales [29, 30, 34] et une lutte biologique [26]. De même, la mise en place des jachères dans le cadre de la réforme de la politique agricole commune a entraîné de nouvelles réflexions sur le statut des mauvaises herbes [9, 31].

Dans le sud-est de la France, où persistent des exploitations agricoles incluant l'élevage ovin itinérant, les fourrages de chaume (éteules) avec leurs communautés d'adventices constituent une biomasse non négligeable en période de soudure (septembre-octobre) et permettent le bouclage de la saison de pâturage [6]. Ce pâturage ovin des champs moissonnés encore appelé vaine pâture était un usage traditionnel très répandu dans toute la France au MoyenAge [27]. Il existait encore dans de nombreuses régions au début du $19^{\mathrm{e}}$ siècle [21]. Aujourd'hui, cette pratique a disparu dans les exploitations intensives (céréaliculture, maïsiculture, etc.) mais elle persiste dans le bassin méditerranéen [35], les régions montagneuses [27] et quelques terroirs aux conditions écologiques marquées [7, 24].

Bien que pratiquée empiriquement par de nombreux éleveurs, peu de données sont disponibles sur la valeur alimentaire des fourrages de champs moissonnés en région Provence-Alpes-Côte-d'Azur (PACA). C'est pourquoi, les objectifs de cette étude sont d'évaluer la valeur nutritive (teneurs minérales) de fourrages issus des champs moissonnés en comparaison avec les autres ressources offertes annuellement aux troupeaux.

\section{MATÉRIEL ET MÉTHODES}

\subsection{Site d'étude}

Les parcelles échantillonnées font partie d'une exploitation agricole située sur la commune de Rustrel $\left(5^{\circ} 26^{\prime} \mathrm{E}, 43^{\circ} 55^{\prime} \mathrm{N}\right)$ au nord de la ville d'Apt, dans le département du Vaucluse, à une altitude avoisinant les 330 mètres. Les massifs et contreforts montagneux de cette région (plateau d'Albion, massif du Luberon) sont caractérisés par des exploitations avec deux activités principales : l'élevage (ovins, caprins) et la polyculture (fourrage, céréales, lavandins, truffières, etc.). L'exploitation agricole choisie est représentative des 81 exploitations en polyculture-élevage du territoire du Parc naturel régional du Luberon (départements du Vaucluse et Alpes-de-Haute-Provence) $[18,25]$. Le circuit de pâturage mis en place durant l'année d'échantillonnage (1999) est stabilisé et ne présente de variations interannuelles qu'en présence de conditions 
climatiques exceptionnelles. Cette exploitation possède encore également une importante flore adventice comprenant de nombreuses espèces rares au niveau national $[10$, 16, 19].

La surface agricole utile de l'exploitation étudiée est de 60 ha dont une surface toujours en herbe de 27 ha. Cette exploitation familiale est de type polyculture-élevage (céréales, luzerne et élevage ovin) avec une seule personne travaillant sur l'exploitation et guidant un troupeau d'ovins de 150 têtes [25]. La conduite du troupeau s'organise en un seul quartier de pâturage mixte (surfaces cultivées et de parcours) regroupé autour de la ferme. Ce quartier comprend des parcelles écologiquement contrastées (pelouses sèches, prairies humides, friches post-culturales) où les parcelles moissonnées sont parcourues en fin du circuit de pâturage journalier de $18 \mathrm{~h}$ à $20 \mathrm{~h}$ entre la mi-juillet et la fin octobre. Parmi les surfaces parcourues, trois parcelles ont été sélectionnées pour le dosage des minéraux de leur végétation. Elles sont chacune représentatives des conditions écologiques et de pâturage des autres parcelles parcourues :

- une friche post-culturale de 1,5 ha. Cette parcelle est abandonnée depuis 5 ans. Sa végétation est dominée par Festuca gr. ovina L. (12,3\%) et Lolium rigidum Gaudin (14,2 \%, Tab. I). Cette parcelle est pâturée par le troupeau d'ovins guidé par l'exploitant et des chiens (pâturage de parcours en troupe) ponctuellement du mois de novembre au mois de mars, plus régulièrement d'avril à octobre ;

Tableau I. Recouvrement spécifique des espèces dominantes (recouvrement $>5 \%$ ) des quatre parcelles étudiées selon la méthode de Daget et Poissonnet [8].

\begin{tabular}{|c|c|c|c|}
\hline Espèces & $\begin{array}{c}\text { Friche } \\
\text { post-culturale }\end{array}$ & $\begin{array}{l}\text { Prairie } \\
\text { humide }\end{array}$ & $\begin{array}{l}\text { Pelouse Parcelle } \\
\text { sèche moissonnée }\end{array}$ \\
\hline
\end{tabular}

$(\%)$

\begin{tabular}{lllll}
\hline Ammi visnaga (L.) Lam. & 0 & 0 & 0 & 5,4 \\
Brachypodium phoenicoides (L.) Roemer \& Schultes & 0,4 & 0 & 39,4 & 0 \\
Convolvulus arvensis L. & 1,7 & 0,5 & 0,6 & 5,0 \\
Coronilla scorpioides (L.) Koch & 0,4 & 0 & 0 & 0 \\
Cynodon dactylon (L.) Pers. & 0 & 16,4 & 0 & 0 \\
Daucus carota L. & 6,1 & 0,5 & 3,7 & 1,0 \\
Festuca arundinacea Schreber & 0,1 & 26,6 & 0 & 0 \\
Galeopsis ladanum subsp. angustifolium & & & & \\
(Hoffman) Celak. & 0 & 0 & 0 & 11,4 \\
Leontodon hispidus L. & 2,9 & 0 & 7,8 & 0 \\
Lolium rigidum Gaudin & 14,2 & 4,6 & 0 & 2,5 \\
Lotus corniculatus L. & 5,7 & 0,1 & 0,6 & 0 \\
Medicago lupulina L. & 4,3 & 11,1 & 4,7 & 0 \\
Polygonum aviculare L. & 0 & 0 & 0 & 42,6 \\
Potentilla reptans L. & 6,6 & 0,5 & 0 & 3,0 \\
Trifolium pratense L. & 2,0 & 7,7 & 0 & 12,4 \\
Vicia cracca L. & 0 & 7,2 & 0,6 & 0 \\
Vicia villosa Roth & 0 & 11,3 & 0,2 & 0 \\
\hline
\end{tabular}


- une prairie humide de 0,3 ha. Seule la partie située le long d'un drain a été échantillonnée car cette parcelle est régulièrement inondée au printemps. En conséquence, elle n'est pas pâturée durant cette période. Par contre, un fauchage mécanique est réalisé à la mi-juin et les animaux pâturent en troupe le regain durant le reste de l'année. Cette prairie est dominée par des Poacées (55\%), dont deux espèces Cynodon dactylon (L.) Pers. (16,4\%), et Festuca arundinacea Schreb. (26,6 \%) sont particulièrement bien représentées, et des dicotylédones où domine une Fabacée Vicia villosa Roth $(11,3 \%)$ (Tab. I) ;

- une pelouse sèche de 1,2 hectare nettement dominée par une Poacée Brachypodium phoenicoides (L.) Roemer et Schultes $(39,4 \%)$ qui présente peu d'intérêt au niveau pastoral. Pour contraindre les ovins à consommer cette espèce, l'exploitant agricole organise sur cette pelouse un pâturage contraint en parc mobile.

La quatrième parcelle est un champ cultivé en blé d'hiver (Triticum aestivum L. var. Darius) d'une surface de 0,4 ha. L'itinéraire technique de cette parcelle est semblable à celui de nombreux champs de cette région [25]. Le labour est effectué à la fin du mois d'octobre $(30 \mathrm{~cm})$ après épandage d'environ 10 tonnes de fumier à l'hectare. Le semis est réalisé début novembre avec $170 \mathrm{~kg}$ de semences par hectare. La parcelle n'est pas irriguée, et elle n'est l'objet ni d'application d'herbicides ni de désherbage mécanique. Elle est moissonnée fin juillet pour un rendement moyen de 35 quintaux/hectare (1996-1999). Après la moisson, les fourrages de chaume et leurs communautés d'adventices sont pâturés jusqu'en octobre (quatre à cinq passages du troupeau par semaine). Les espèces qui dominent cette communauté d'adventices en juillet 1999 sont Polygonum aviculare L. (42,6 \%) et Trifolium pratense L. (12,4\%) [10].

\section{2. Échantillonnage des fourrages}

Dans chaque parcelle, trois exclos d'une surface de $4 \mathrm{~m}^{2}(2 \times 2 \mathrm{~m})$ ont été installés de façon à tenir compte de l'hétérogénéité des groupements végétaux présents. Ces exclos, d'une hauteur de grillage de 1 mètre, ont pour but d'éviter le piétinement dû aux passages du troupeau qui est responsable de biais dans les analyses minérales par enrichissement des surfaces foliaires en terre [23]. Dans chaque exclos, la biomasse est échantillonnée en coupant les végétaux à $3 \mathrm{~cm}$ du sol dans des placettes de $2500 \mathrm{~cm}^{2}$ $(50 \times 50 \mathrm{~cm})$. Chaque prélèvement est effectué deux fois par mois entre mai et octobre 1999, pour la friche post-culturale, la prairie humide et la pelouse sèche. Pour le champ de céréales, les prélèvements sont effectués selon la même méthodologie mais après la moisson, de juillet à octobre 1999. Chaque prélèvement de placettes $(50 \times 50 \mathrm{~cm})$ est réalisé de façon aléatoire dans les exclos, mais aucune placette n'est échantillonnée deux fois au même endroit durant la campagne d'échantillonnage. À la fin de la saison de végétation, 36 prélèvements ont été effectués dans chacun des milieux friche post-culturale, prairie humide, pelouse sèche et 24 prélèvements dans le champ de céréales, soit un total de 132 prélèvements.

\subsection{Traitements et analyse minérale des échantillons}

Après la coupe, les prélèvements sont pesés directement sur le terrain (poids frais). Ils sont ensuite placés dans des sacs puis transportés au laboratoire pour être trempés dans de l'eau distillée et lavés d'éventuelles contaminations par de la terre. Les échantillons sont ensuite placés en étuve (environ 24 heures à $50{ }^{\circ} \mathrm{C}$ ) jusqu'à obtention d'un poids constant (poids sec).

Les échantillons de fourrage sont moulus au moulin à marteaux puis homogénéisés au broyeur «Cyclotec » avec une grille de $2 \mathrm{~mm}$. La poudre d'échantillon est conservée dans des pots en plastique hermétiques à l'abri de l'humidité. La teneur en azote total exprimée en matière azotée totale (MAT) des échantillons est mesurée par la méthode Kjeldahl (minéralisation au « Tecator 1015 » 
et distillation au « Tecator $1003 »)$. La cellulose brute (Cell.) est dosée par la méthode de Weende adaptée au Fibertec.

Les échantillons sont minéralisés par calcination à $450{ }^{\circ} \mathrm{C}$ et les cendres totales (CT) sont reprises à l'acide nitrique concentré. Après lavage et filtration, les cendres insolubles (CI) sont quantifiées par gravimétrie. Sur le filtrat complété à $100 \mathrm{~cm}^{3}$ pour les éléments majeurs ( $\mathrm{K}, \mathrm{P}, \mathrm{Na}, \mathrm{Ca}, \mathrm{Mg}$ ) et $50 \mathrm{~cm}^{3}$ pour les oligo-éléments $(\mathrm{Fe}, \mathrm{Cu}, \mathrm{Zn}$, $\mathrm{Mn}$ ), les cendres solubles (CS) sont dosées, le phosphore par colorimétrie au métavanadate d'ammonium et les autres éléments par spectrophotométrie d'absorption atomique [11-14, 23].

\subsection{Caractérisation de la flore des exclos}

En juin 1999, l'analyse quantitative de la composition floristique a été réalisée grâce à des relevés transects [8], méthode adaptée à l'observation de modifications fines dans la structure des communautés herbacées (changement en fréquence du recouvrement des espèces). Pour chaque parcelle, 300 points de lecture sont effectués (Tab. I). Des visites complémentaires sont effectuées pour identifier les espèces qui fleurissent plus tardivement dans l'année.

\subsection{Analyses statistiques}

Une Analyse en Composantes Principales (ACP) a été réalisée sur la matrice des 12 analyses ( 9 éléments minéraux, matière azotée totale, cellulose et cendres insolubles) pour 22 valeurs mensuelles (6 [mai à octobre] pour la friche post-culturale, la prairie humide, la pelouse sèche et 4 [juillet à octobre] pour la parcelle moissonnée). Chacune de ces 22 valeurs mensuelles est la moyenne de dosage de 6 échantillons ( 3 prélèvements par parcelle échantillonnés bimensuellement).

Afin de dégager des différences significatives entre les parcelles et/ou les dates de prélèvement, un test ANOVA global est effectué sur l'ensemble des valeurs mensuelles pour la période estivale (juilletoctobre). Si le test ANOVA global indique un effet significatif entre les parcelles, entre les dates de prélèvements ou entre ces deux facteurs combinés, des comparaisons de moyennes a posteriori (test de Fisher) sont ensuite réalisées. Toutes les analyses statistiques sont effectuées grâce au logiciel STATVIEW [32].

\section{RÉSULTATS}

\subsection{Analyse en composantes principales (ACP) et classification hiérarchique ascendante (CHA)}

Les deux premiers axes de l'ACP réalisée sur la matrice des 12 analyses et 22 échantillons expliquent $78 \%$ de la variance totale avec respectivement $40 \%$ pour l'axe 1 et $38 \%$ pour l'axe 2 (Figs. 1a et $1 \mathrm{~b}$ ). L'axe 1 oppose, sur la totalité de la période d'échantillonnage, le fourrage de la parcelle moissonnée, particulièrement corrélé avec les plus fortes teneurs mesurées en $\mathrm{Mg}$ (contribution : $19,3 \%), \mathrm{Ca}(14,9 \%)$ et MAT $(11,9 \%)$, aux autres parcelles plutôt corrélées avec de fortes teneurs mesurées en Cell. $(8,8 \%)$ et le $\mathrm{Zn}(7,8 \%)$. De même, l'axe 2 oppose, le fourrage de la parcelle moissonnée, corrélé avec de fortes teneurs mesurées en Fe (16,9\%), Cu (13,8\%) et Mn (12,2\%) à la parcelle de prairie humide plutôt corrélée avec de fortes teneurs en $\mathrm{P}(13,8 \%)$ et $\mathrm{K}(9,8 \%)$.

La CHA réalisée sur la même matrice permet de regrouper, pour l'ensemble des analyses, les valeurs obtenues entre juillet et octobre pour les fourrages de chaume avec les adventices et, entre mai et octobre pour les 3 autres parcelles. Les résultats de cette CHA permettent de regrouper les résultats d'une même parcelle, qui est alors entourée d'une ellipse (Fig. 1b). Des flèches permettent de visualiser une dynamique temporelle au sein de chaque parcelle. Ainsi, de 
(a)

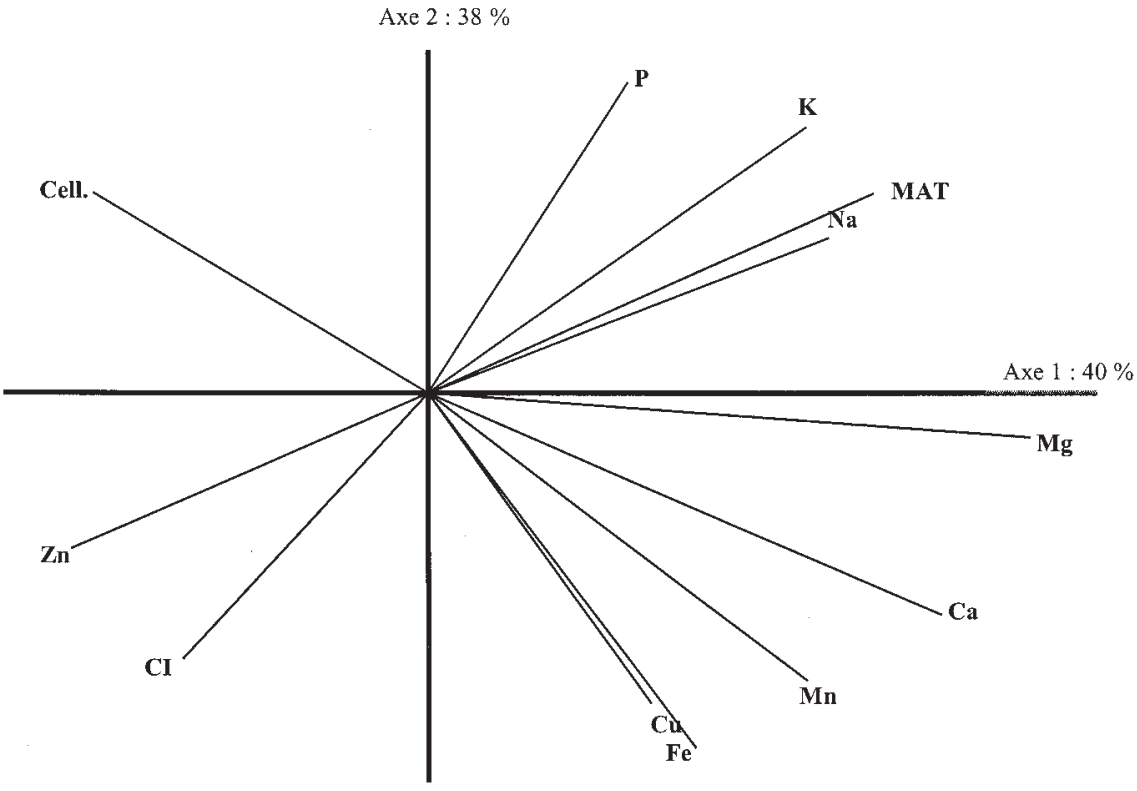

(b)

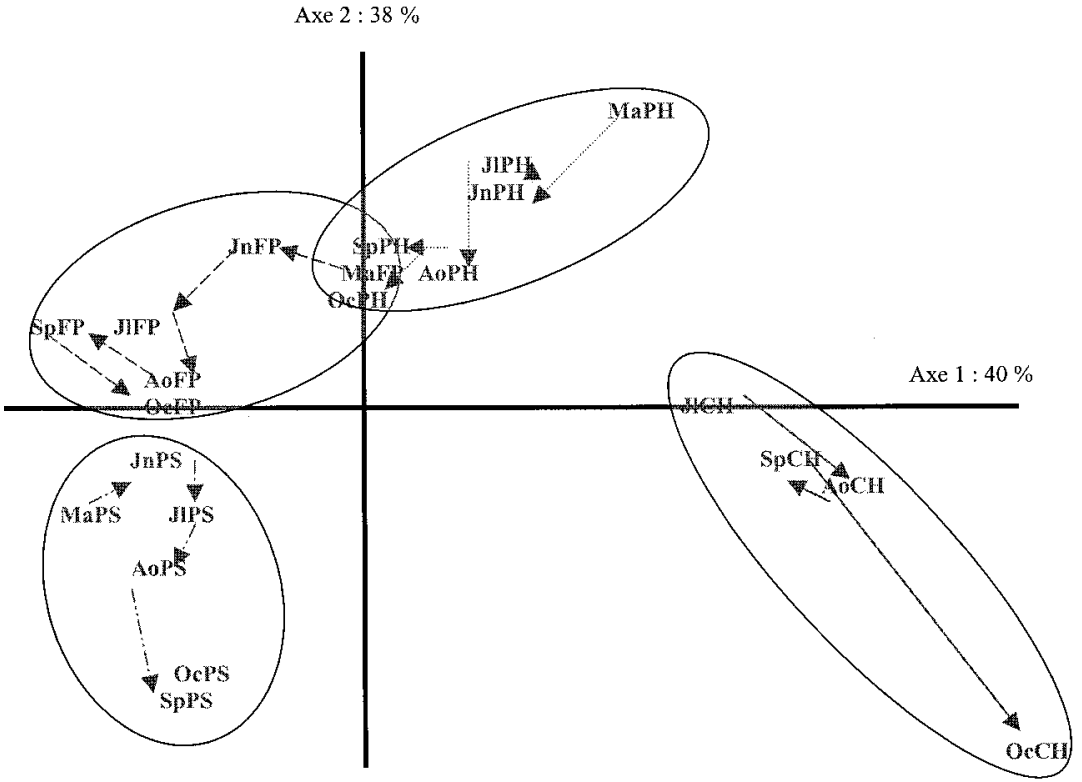

Figure 1. Analyse en composante principale (ACP) réalisée sur la matrice des 12 analyses, ( 9 éléments minéraux, matière azotée totale, cellulose et cendres insolubles) pour 22 valeurs mensuelles (6 [mai à octobre] pour la friche post-culturale, la prairie humide, la pelouse sèche et 4 [juillet à octobre] pour la parcelle moissonnée). Chacune de ces 22 valeurs mensuelles est la moyenne de dosage de 6 échantillons ( 3 prélèvements par parcelle échantillonnée bi-mensuellement). 2 (a) cercle des corrélations, 2 (b) analyse en composantes principales. $(\mathrm{Ma}=$ mai ; Jn $=$ juin ; $\mathrm{Jl}=$ juillet ; $\mathrm{Ao}=$ août $; \mathrm{Sp}=$ septembre $; \mathrm{Oc}=$ octobre $; \mathrm{PH}=$ prairie humide $; \mathrm{FP}=$ friche post-culturale ; $\mathrm{PS}=$ pelouse sèche $; \mathrm{CH}=$ fourrage de chaume). 
juillet à octobre, les valeurs mesurées pour le fourrage de la parcelle moissonnée se déplacent vers la droite, le long de l'axe 1 ce qui traduit une augmentation des teneurs en Mn, Fe et $\mathrm{Cu}$. Au contraire, les valeurs mesurées pour les 3 autres parcelles se déplacent vers le bas de l'axe 2, traduisant une diminution des teneurs en MAT, mais une augmentation des teneurs en Cell. et en CI, sensible surtout pour la parcelle en pelouse sèche.

\subsection{Comparaisons de moyennes}

Les résultats du test global portant sur les valeurs des mois de juillet à octobre montrent que le fourrage échantillonné dans la parcelle moissonnée est significativement moins abondant que dans les autres parcelles. Il présente des teneurs inférieures en cellulose $(P<0,0001)$, cendres insolubles $(P<0,001)$ et zinc $(P<0,01)$, mais ses teneurs sont significativement supérieures en $\mathrm{Mg}, \mathrm{Ca}, \mathrm{K}$ et MAT $(P<0,0001)$; Mn $(P<0,001), \mathrm{Fe}(P<0,01)$ et $\mathrm{Cu}(P<0,01)$. Aucune variation significative n'est à noter pour $\mathrm{P}$ et $\mathrm{Na}$.

Afin de préciser ces résultats, des comparaisons de moyennes sont aussi réalisées entre le fourrage échantillonné dans la parcelle moissonnée et dans chacune des trois autres parcelles du parcours (Fig. 2). Les résultats confirment que le fourrage échantillonné dans la parcelle moissonnée possède toujours une biomasse sèche $(P<0,0001)$ et des teneurs en cellulose $(P<0,0001)$ significativement inférieures à celles des autres parcelles. Au contraire, le fourrage de chaume présente des teneurs significativement supérieures pour $\mathrm{Mg}$ $(P<0,0001), \mathrm{Ca}(P<0,0001$ pour la friche post-culturale et la prairie humide et $P<0,0001$ par rapport à la prairie sèche) et Mn (respectivement $P<0,01 ; P<0,001$ et $P<0,05$ par rapport aux parcelles en friche post-culturale, prairie humide et pelouse sèche). Pour K et MAT $(P<0,0001)$, ces valeurs ne deviennent significativement supérieures que par rapport à la friche post-culturale et la pelouse sèche. Pour l'élément $\mathrm{Na}$, les mêmes variations sont observées que pour K et MAT mais avec des seuils inférieurs (respectivement pour la friche post-culturale et la prairie sèche $P<0,05$ et $P<0,01)$. Pour le Cu $(P<0,01)$ et le Fe $(P<0,001)$, les valeurs restent significativement supérieures uniquement par rapport à la friche post-culturale et la prairie humide. Pour l'élément $\mathrm{Zn}$ ainsi que les CI, le fourrage échantillonné dans la parcelle moissonnée est significativement plus faible pour ces variables exclusivement par rapport à la pelouse sèche au seuil $P<0,001$. Pour l'élément $\mathrm{P}$, le fourrage échantillonné dans la parcelle moissonnée est significativement inférieur à celui échantillonné dans la prairie humide $(P<0,001)$ mais supérieur à celui échantillonné dans la pelouse sèche $(P<0,01)$.

\section{DISCUSSION}

Pour un végétal donné, Jarrige [22] montre que les relations entre cellulose et lignine sont constantes. Or, la lignine n'est pas digestible et fait obstacle à la digestion des glucides par les microbes de la panse. De même, les cendres insolubles sont composées en majorité de silice qui est un élément peu fixé par l'organisme. Les fourrages de chaume et de leurs adventices constitueront donc un aliment plus facile à digérer par les ovins car ils sont significativement plus pauvres pour ces deux éléments. Ces faibles teneurs peuvent s'expliquer par la composition floristique de la communauté d'adventices présente dans la parcelle moissonnée. En effet, ce fourrage est composé d'une majorité de dicotylédones ( $86 \%$ ) alors que la composition floristique des 3 autres parcelles est dominée par des monocotylédones dont la richesse en cellulose et cendres insolubles est bien connue [12, 13].

Quant au zinc, les teneurs mesurées pour la parcelle moissonnée apparaissent comme les plus faibles et au dessous du seuil de carence animale, fixé pour les ovins, à 

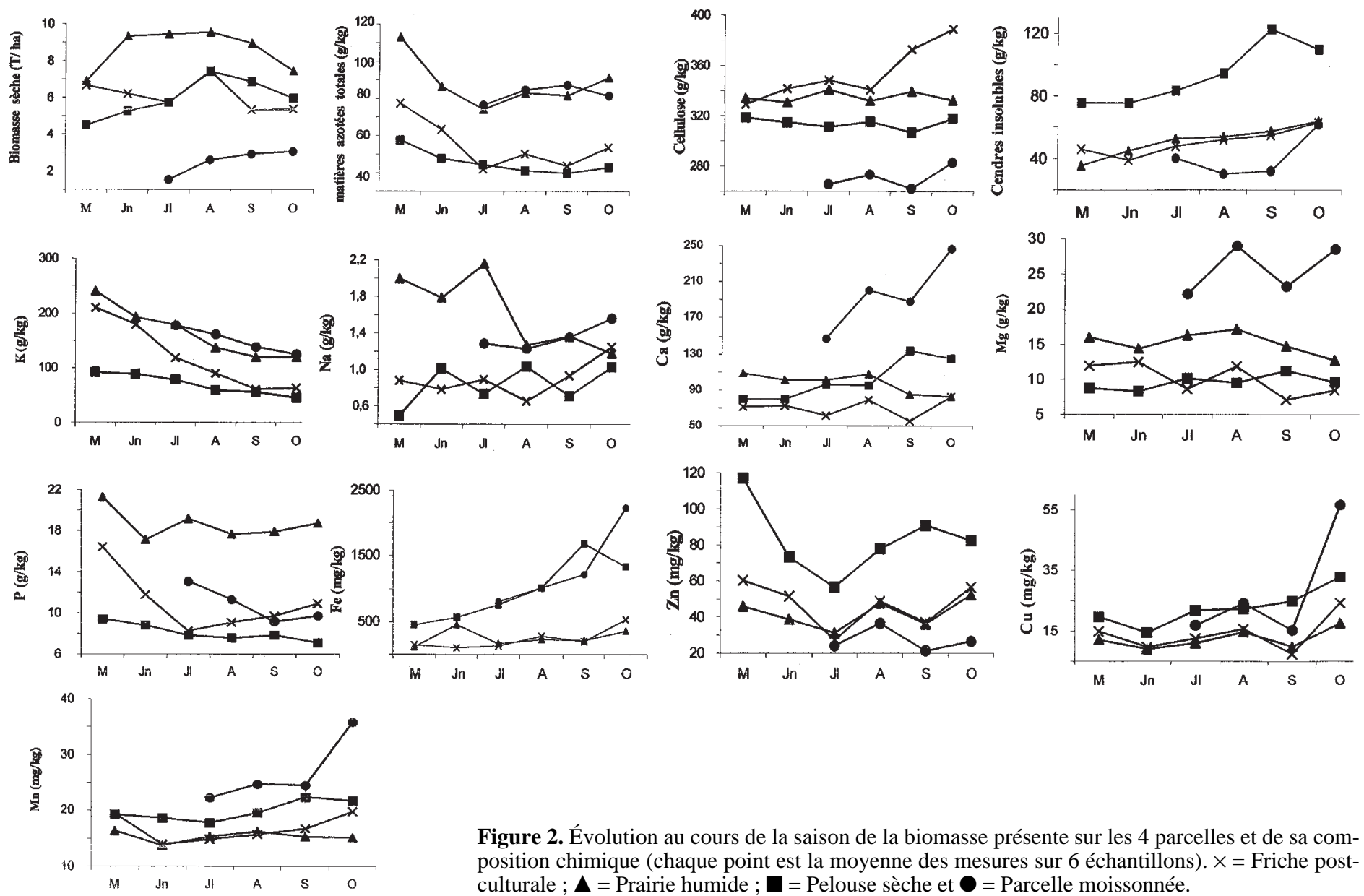

Figure 2. Évolution au cours de la saison de la biomasse présente sur les 4 parcelles et de sa composition chimique (chaque point est la moyenne des mesures sur 6 échantillons). $\times=$ Friche postculturale $; \boldsymbol{\Lambda}$ = Prairie humide $; \boldsymbol{\square}=$ Pelouse sèche et $\boldsymbol{0}=$ Parcelle moissonnée 
$45 \mathrm{ppm}$ [22]. Ces faibles teneurs en zinc sont cependant caractéristiques des herbages sur sol calcaire [14]. Les fourrages échantillonnés dans les 3 autres parcelles possèdent cependant des teneurs en zinc significativement supérieures à la parcelle moissonnée et aux seuils de carence. La pratique du pâturage de parcours permet d'organiser journellement la ration alimentaire avec des passages dans les parcelles moissonnées incluses dans le circuit de pâturage (18 h-20 h en fin de circuit). La complémentarité des ressources herbagères permet alors de pallier les carences en zinc de ce fourrage.

Ces carences pourraient aussi être facilement palliées par l'apport de compléments minéraux et vitaminiques (CMV). Cependant, dans un système d'élevage extensif basé sur le pâturage de parcours, il est nécessaire d'optimiser la complémentarité de ressources fourragères hétérogènes en liaison avec des situations environnementales contrastées [11]. Chaque ressource doit ainsi être exploitée en correspondance avec les besoins physiologiques du troupeau durant une période spécifique [20]. Dans notre étude, l'évolution des teneurs minérales dosées dans la parcelle moissonnée et les trois autres parcelles évoluent de manière contrastée entre juillet et octobre 1999. Ces teneurs ont tendance à augmenter au cours de la campagne d'échantillonnage pour la parcelle moissonnée à l'inverse des trois autres parcelles. Ainsi, des teneurs significativement plus élevées en Matières Azotées Totales, calcium, magnésium, cuivre, sodium et potassium durant les mois de septembre et d'octobre permettent d'affirmer que le pâturage de la parcelle moissonnée fournit un apport en éléments minéraux essentiel en période de soudure automnale [6]. En effet, les autres parcelles parcourues au sein de l'exploitation sont peu intéressantes au niveau pastoral durant cette période et le pâturage des parcelles moissonnées permet alors à l'agriculteur de ne pas compléter la ration des ovins avec des fourrages importés. En octobre, même si la biomasse des chaumes et de leurs adventices demeure significativement inférieure à celles des autres parcelles échantillonnées, sa qualité lui permet de pallier les carences et la faible digestibilité des ressources herbagères disponibles sur l'exploitation à la même époque.

Les objectifs de cette étude ne sont pas de proposer le pâturage des parcelles moissonnées comme substitutif du parcours mais bien de démontrer que cette pratique multiséculaire permet de compléter qualitativement la ration alimentaire des ovins surtout en période de soudure, quand les autres ressources herbagères du quartier de pâturage sont qualitativement moins intéressantes. L'existence dans les parcelles moissonnées d'une communauté d'adventices riche en espèces apparaît alors pour l'agriculteur pratiquant le pâturage de parcours en région méditerranéenne, comme une ressource à pérenniser par une réduction des traitements herbicides et de l'application d'engrais azotés qui favorisent plus les espèces de la famille des Poacées que les dicotylédones [33].

\section{REMERCIEMENTS}

Nous sommes reconnaissants au Professeur Thierry Tatoni et au Docteur Laurence Affre pour leurs aides lors des traitements statistiques ainsi qu'à Christian Audoynaud, Daniel et Isabelle Wagnies, Jean Gerbaud et Delphine Auvré pour leur aide technique. Nous remercions le Parc Naturel Régional du Luberon et Jean-Louis Armand pour leur autorisation d'accès aux sites lors des campagnes de terrain. Nos remerciements vont également à l'ensemble du personnel du laboratoire d'écologie des prairies de Michamps en Belgique pour leur accueil et leurs aides diverses lors de la réalisation des analyses minérales. Nous remercions aussi deux lecteurs anonymes pour leurs corrections qui ont permis d'améliorer une première version du texte.

\section{RÉFÉRENCES}

[1] Albrecht H., Changes in the arable weed flora of Germany during the last fives decades, in: Proceedings of the 9th EWRS Symposium, Budapest, Hongrie, 1995, pp. 41-48. 
[2] Andraesen C., Stryhn H., Streibig J.C., Decline of the flora in Danish arable fields, J. Appl. Ecol. 33 (1996) 619-626.

[3] Aymonin G., Les messicoles vont-elles disparaître ?, Science et Nature 49 (1962) 3-9.

[4] Aymonin G., Origine présumée et disparition progressive des « adventices messicoles calcicoles » en France, in: $\mathrm{II}^{\mathrm{e}}$ colloque sur la biologie des mauvaises herbes, ENSAG, France, 1965, p. 10 .

[5] Aymonin G., Observation sur le processus de régression des adventices des cultures ( $\ll$ mauvaises herbes ») et processus biocénologiques, in: $\mathrm{IV}^{\mathrm{e}}$ colloque international sur l'écologie et la biologie des mauvaises herbes, Marseille, France, 1973, p. 10

[6] Bellon S., Rôles des adventices dans le pâturage ovin en région méditerranéenne, in: Dalmas J.P. (Ed.), Faut-il sauver les mauvaises herbes ?, Conservatoire Botanique National de Gap-Charance, France, 1997, pp. 159-165.

[7] Benoit M., Laignel G., Liénard G., Dedieu B., Chabosseau J.M., Eléments de réussite économique des élevages ovins extensifs du Montmorillonnais, Ann. Zootech. 47 (1998) 465-473.

[8] Daget P., Poissonet J., Une méthode d'analyse phytologique des prairies, Ann. Agron. 22 (1971) 5-41.

[9] Dekker J., Weed diversity and weed management, Weed Sci. 45 (1997) 357-363.

[10] Dutoit T., Gerbaud E., Ourcival J.M., Field boundary effects on soil seed banks and weed vegetation distribution in an arable field withou weed control (Vaucluse, France), Agronomie 19 (1999) 579-590.

[11] Dutoit T., Le pâturage itinérant dans la basse vallée de la Seine (France) : une nécessité écologique et agronomique, Cah. Agric. 8 (1999) 486-497.

[12] Dutoit T., Alard D., Mineral contents of chalk grasslands in relation with sheep grazing involved in conservation management systems, Rev. Ecol. Terre \& Vie 52 (1997) 9-20.

[13] Dutoit T., Alard D., Mineral content and plan diversity in chalk grassland under different management: a case study in Normandy, France, in West N.E. (Ed.), Rangelands in a sustainable biosphere, Society for Range Management, Denver, Colorado, USA, 1995, pp. 122-123.

[14] Dutoit T., Alard D., Lambert J., Frileux P.N. Biodiversité et valeur agronomique des pelouses calcicoles : effets du pâturage ovin, Fourrages 142 (1995) 145-158.

[15] Dutoit T., Gerbaud E., Ourcival J.M., Roux M. Alard D., Recherche prospective sur la dualité entre caractéristiques morphologiques et capacités de compétition des végétaux : le cas des espèces adventices et du blé, C. R. Acad. Sci., Série III, 324 (2001) 1-12.
[16] Filosa D., Situation de quelques espèces végétales messicoles en Haute-Provence occidentale, Bull. Soc. Bot. Centre-Ouest 16 (1985) 61-77.

[17] Firbank L.G., Interactions between weeds and crops, in: Firbank L.G., Carter N., Darbyshire J.F., Potts G.R. (Eds.), The ecology of temperate cereal fields, Blackwell Scientific Publications, Oxford, UK, 1991, pp. 209-231.

[18] Garcia F., Lasseur J., Leouffre M.C., Diversité de l'élevage ovin en Luberon, Suivi scientifique de l'opération «pelouses sèches calcaires », INRA, Avignon, France, 2000, 16 p.

[19] Guende G., Olivier L., Les mesures de sauvegarde et de gestion des plantes messicoles du Parc Naturel Régional du Luberon, in: Dalmas J.P. (Ed.), Faut-il sauver les mauvaises herbes ?, Conservatoire Botanique National de GapCharance, France, 1997, pp. 179-187.

[20] Guerin G., Leger F., Pflimlin A., Stratégie d'alimentation - Méthodologie d'analyse et de diagnostic de l'utilisation et de la gestion des surfaces fourragères et pastorales, Institut de l'élevage, Paris, 1994.

[21] Hubert B., Deverre C., Meuret M., Les combats des parcours, Réussir Pâtre 445 (1997) 16-18.

[22] Jarrige R., Alimentation des bovins, ovins et Caprins, INRA (Eds.), Versailles, France, 1988.

[23] Lambert J., Modes opératoires : analyse des végétaux, Laboratoire d'écologie des prairies, Université Catholique de Louvain, Michamps, Belgique, 1992.

[24] Luick R., Ecological and socio-economic implications of livestock-keeping systems on extensive grassland in south-western germany, J. Appl. Ecol. 35 (1998) 979-982.

[25] Mahieu P., Suivi agronomique d'une opération locale agri-environnementale de protection in situ des plantes messicoles dans le Luberon, Mémoire de DAA agri-environnement, IMEPENSA, Montpellier, France, 1997.

[26] McFadyen R.E.C., Biological control of weeds, Ann. Rev. Entomol. 43 (1998) 369-393.

[27] Meuret M., Bellon S., Guérin G., Hanus G., Faire pâturer sur parcours, Renc. Rech. Ruminants 2 (1995) 27-36.

[28] Montegut J., Évolution et régression des messicoles, in: Dalmas J.P. (Ed.), Faut-il sauver les mauvaises herbes ?, Conservatoire Botanique National de Gap-Charance, France, 1997, pp. 11-32.

[29] Mortensen D.A., Bastiaans L., Sattin M., The role of ecology in the development of weed management systems: an outlook, Weed Res. 40 (2000) 49-62.

[30] Ovenden G.Y., Swash A.R.H., Smallshire D., Agri-environment schemes and their contribution to the conservation of biodiversity in England, J. Appl. Ecol. 35 (1998) 955-960. 
[31] Rodriguez A., Mamarot J., La jachère dans les rotations : Étude des risques de salissement des terres, ACTA Point n 2, Paris, France, 1994.

[32] Statview pour Windows, version 4.55, Abacus Concept, Inc., Berkeley, CA, USA, 1992-1997.

[33] Sutcliffe O.L., Kay Q.O.N., Changes in the arable flora of central southern England since the 1960s, Biol. Cons. 93 (2000) 1-8.

[34] Verdier J.L., Desecures J.P., Mamarot J., Influence du travail du sol et du désherbage sur l'évolution de la flore adventice de surface, Columa 1 (1992) 171-184.
[35] Verlaque R., Filosa D., Caryologie et biogéographie des messicoles menacées du sud-est de la France (comparaison avec les autres mauvaises herbes), in: Dalmas J.P. (Ed.), Faut-il sauver les mauvaises herbes?, Conservatoire Botanique National de Gap-Charance, France, 1997, pp. 105-124.

[36] Wilson P.J., Conserving the flora of arable field margins - How much does it cost?, in: Brighton Crop Conference Protection - Weeds, Brighton, UK, 1997, pp. 991-996. 